\title{
Giant disk galaxies : Where environment trumps mass in galaxy evolution
}

\author{
H. M. Courtois \\ University of Lyon 1 - CNRS/IN2P3, IPNL 4 rue Enrico Fermi 69622 Villeurbanne, France \\ email: h.courtois@ipnl.in2p3.fr
}

\section{Direct evidence for environmentally driven galaxy evolution}

There is an ongoing argument regarding galaxies, like there is regarding children, of whether the final outcome is driven primarily by nature or nurture. In the case of galaxies, the total mass plays the role of genetics (nature) and the number of nearby galaxies plays the role of family life (nurture). Untangling the role of each has been particularly difficult for galaxies because the mass of a galaxy is closely tied to its environment.

By combining data from the Cosmic Flows project, which has consistently reanalyzed archival galaxy HI profiles, and 3.6 $\mathrm{m}$ photometry obtained with the Spitzer Space Telescope, with which we can measure stellar mass, we use the baryonic Tully-Fisher (BTF) relationship to explore whether these massive galaxies are distinct (Zaritsky et al. 2014, Courtois et al. 2015). We discuss several results, but the most striking is the systematic offset of the HI-massive sample above the BTF. These galaxies have both more gas and more stars in their discs than the typical disc galaxy of similar rotational velocity.

We were able to identify very massive galaxies that live in isolated environments, enabling us to break the usual connection between mass and environment. We find that these galaxies have the properties connected usually with galaxies in poor environments rather than with large masses - thereby showing that (at least in these cases) the dominant influence was environment (nurture) rather than mass (nature).

It is found that the HI-massive galaxies lie systematically above the Baryonic TullyFisher (BTF). If these deviations are interpreted as variations in the condensed baryon fraction $f_{C}$, then we conclude that there are galaxies in which $f_{C}$ approaches 1 , or in other words the entire allotment of baryons for that halo have found their way to the disc. In spite of this, or whatever other process may be driving the deviation from the BTF, the star formation efficiency, when quantified as the fraction of disc baryons that are in stars, is the same in these systems as in more typical galaxies.

We conclude that the star formation efficiency is driven by internal self-regulation, while in the condensed baryon fraction $f_{C}$ can be altered by external factors.

We find environment affects the condensation of baryons onto the central galaxy but not the fraction of those that are converted to stars. When looking in detail for environmental differences, we found that the most massive HI detected galaxies are located preferentially in filaments. We present the first evidence of an environmental effect on galaxy evolution using a dynamical definition of a filament.

\section{References}

Courtois, H. et al. 2015, MNRAS, 448, 1767

Zaritsky, D. et al. 2014, AJ, 147, 134 